\title{
CHEMICAL ABUNDANCES FROM PLANETARY NEBULAE IN
}

\section{THE MAGELLANIC CLOUDS}

\author{
M. J. BARLOW \\ Department of Physics \& Astronomy, University College London, \\ Gower Street, London WC1E 6BT, U.K.
}

\begin{abstract}
.
Heavy element abundances, in particular those of oxygen, obtained from recent spectroscopic surveys of Magellanic Cloud planetary nebulae (PN), are reviewed and compared with those derived for $\mathrm{H}$ regions and objects in our own galaxy. These abundances have been based on collisionally excited lines and are very sensitive to the adopted electron temperature. There is increasing evidence that temperature or density fluctuations within nebulae lead to the electron temperatures being overestimated, with the corollary that the heavy element abundances have been underestimated.
\end{abstract}

Since the mid-1980's, a number of large-scale spectroscopic studies of Magellanic Cloud PN have been carried out at optical wavelengths, with several making use of IUE ultraviolet spectra as well. At optical wavelengths, Monk et al. (1988) studied 71 SMC and LMC PN, Boroson et al. (1989) and Henry et al. (1989) studied 29 very faint Magellanic Cloud PN, Meatheringham \& Dopita (1991a,b) and Dopita \& Meatheringham (1991a,b) studied 82 PN, Vassiliadis et al. (1992) observed $~ 40$ faint PN, Jacoby \& Kaler (1993) observed 12 very faint PN, and de Freitas Pacheco et al. (1993a,b) observed 8 Type I and 15 non-Type I PN in the LMC. In studies utilising both optical and ultraviolet data, Aller et al. (1987) observed 12 LMC PN and Walton et al. (1991; see also Barlow 1991) studied 43 PN. All of the above studies derived heavy element abundances from analyses of collisionally excited nebular lines. In addition, analyses of various combinations of some the above data have been published by Henry $(1989,1990)$, Kaler \& Jacoby $(1990,1991)$ and Richer (1993). Table I summarises oxygen abundances derived for LMC and SMC PN and (for comparison) for $\mathrm{H}$ II regions in the same Galaxy. The solar oxygen abundance and the abundance of oxygen in solar neighborhood PN and $\mathrm{H}$ II regions, are also listed in Table I. Current evolutionary models for the progenitor stars of PN predict that there should be no significant modification to the original oxygen abundance during their evolution and this is confirmed by the results in Table I, which show the $\mathrm{PN}$ and $\mathrm{H}$ II regions in each galaxy to have the same oxygen abundance. Note that the Type I PN also have the same oxygen abundance as the other nebulae. There is no evidence for significant depletion of oxygen by the CNO-cycle in the Type I PN (believed to be descended from stars at the upper end of the initial mass distribution that produces PN), in agreement with theoretical predictions (Becker \& Iben 1980). Table I shows the O-abundance in the LMC to be 0.3 dex lower than in the Milky Way. The SMC results show a larger scatter than do the LMC results, but are consistent with an average $\mathrm{O}$-abundance in the $\mathrm{SMC}$ which is 0.28 dex lower than that of the LMC.

In recent years increasing doubt has been cast on the accuracy of heavy element abundances derived fron collisionally excited nebular lines. This has its origin in the proposal by Peimbert $(1967,1971)$ that significant temperature fluctuations must exist within ionized nebulae (defined by the ' $t$ ' ${ }^{2}$ parameter), in order to ex- 
TABLE I

Galactic and Magellanic Cloud oxygen abundances from forbidden-line analyses

\begin{tabular}{lccl}
\hline Object Type & $\log (\mathrm{O} / \mathrm{H})+12.0$ & No. of objects & Reference \\
\hline Sun & & & \\
& $8.87 \pm 0.07$ & 1 & Grevesse \& Noels (1993) \\
Gal. H & & & \\
Gal. PN & $8.70 \pm 0.04$ & 22 & Shaver et al. (1983) \\
Gal. PN (Type I) & $8.69 \pm 0.15$ & 42 & Kingsburgh \& Barlow (1994) \\
& $8.65 \pm 0.15$ & 11 & Kingsburgh \& Barlow (1994) \\
LMC H & & & \\
LMC H & $8.39 \pm 0.12$ & 20 & Pagel et al. (1978) \\
LMC H & $8.38 \pm 0.08$ & 4 & Dufour et al. (1982) \\
LMC PN & $8.37 \pm 0.22$ & 4 & Russell \& Dopita (1990) \\
LMC PN & $8.49 \pm 0.15$ & 32 & Monk et al. (1988) \\
LMC PN & $8.30 \pm 0.06$ & 27 & Dopita \& Meatheringham (1991a) \\
LMC PN (Type I) & $8.41 \pm 0.18$ & 12 & Walton et al. (1991), Barlow (1991) \\
LMC PN & $8.41 \pm 0.20$ & 5 & Walton et al. (1991), Barlow (1991) \\
LMC PN (Type I) & $8.42 \pm 0.25$ & 15 & de Freitas Pacheco et al. (1993b) \\
SMC H & & 8 & de Freitas Pacheco et al. (1993a) \\
SMC H & $7.98 \pm 0.05$ & 23 & Pagel et al. (1978) \\
SMC H & $8.02 \pm 0.08$ & 3 & Dufour et al. (1982) \\
SMC PN & $8.13 \pm 0.10$ & 6 & Russell \& Dopita (1990) \\
SMC PN & $8.11 \pm 0.17$ & 7 & Aller et al. (1981) \\
SMC PN & $8.26 \pm 0.15$ & 13 & Monk et al. (1988) \\
SMC PN & $8.08 \pm 0.08$ & 11 & Dopita \& Meatheringham (1991a) \\
& $8.24 \pm 0.13$ & 14 & Walton et al. (1991), Barlow (1991) \\
\hline
\end{tabular}

plain phenomena such as Balmer-jump hydrogen recombination temperatures being systematically lower than [O III] forbidden-line temperatures. In such cases, higher-excitation transitions such as [O III] $\lambda 4363$ would be preferentially excited in the hottest regions, so that the electron temperature $T_{e}$ derived from the [ $O$ iII] 4363/5007 ratio would be too high and thus the abundances derived from the forbidden lines (depending exponentially on the adopted $\mathrm{T}_{e}$ ) would be too low. The implication is that if such temperature fluctuations are present in nebulae then it is better to use recombination lines to derive heavy-element abundances, since the strengths of recombination lines have a much weaker sensitivity to temperature than do those of collisionally excited lines. In fact, since heavy-element recombination lines have a similar power-law sensitivity to temperature as Balmer recombination lines such as $H \beta$, and have no significant dependence on density, the resulting $\mathrm{X}^{n+} / \mathrm{H}^{+}$abundance ratios are virtually independent of the adopted electron temperature and density. 
Peimbert, Storey \& Torres-Peimbert (1993) used O II recombination lines (adopting LS-coupling recombination coefficients) to measure $\mathrm{O} / \mathrm{H}$ ratios for two $\mathrm{H}$ II regions and one $\mathrm{PN}$ in our Galaxy. Relative to the $\mathrm{O} / \mathrm{H}$ ratios derived from forbidden lines, the recombination lines yielded $\mathrm{O} / \mathrm{H}$ ratios that were $0.25-0.30$ dex larger. Liu et al. (1995) and Barlow et al. (1995) have derived O/H ratios for 7 bright Galactic PN from O II recombination lines, using new Intermediate Coupling recombination coefficients that yielded excellent agreement between dozens of $\mathrm{O}$ II lines. The resulting $\mathrm{O} / \mathrm{H}$ ratios were found to be on average 2-3 times larger than those derived from forbidden line analyses and in one extreme case (NGC 7009, Liu et al. 1995) were a factor of 5 larger! Unfortunately, the fainter apparent flux levels of Magellanic Cloud nebulae makes observations of the relatively weak $\mathrm{O}$ II recombination lines much more difficult for them. However, the implication is clear: the abundances listed in Tables 1 and 2, from forbidden line analyses, may be systematically lower than the 'real' abundances. However, it is expected that relative abundances derived from collisonally excited lines should be more accurate, e.g. $\mathrm{N} / \mathrm{O}, \mathrm{C} / \mathrm{N}$ ratios, etc., should be reliable, as should the derived differences in heavy element abundances (e.g. $\mathrm{O} / \mathrm{H}$ ) between one galaxy and another.

The physical origin of the implied temperature fluctuations within $\mathrm{H}$ II regions and PN is not clear at present. Peimbert et al. (1992) have discussed possible mechanisms, including (a) abundance variations, or gradients, within nebulae and (b) shock excitation (caused e.g. by a stellar wind colliding with the nebula) giving rise to hot dense regions which could preferentially excite [O III] $\lambda 4363$ compared to $\lambda \lambda 5007,4959$. Another possibility, explored by Viegas \& Clegg (1994) is that the effect is not due to temperature fluctuations at all, but is instead due to strong density fluctuations within the nebulae. Condensations with high densities $\left(\mathrm{n}_{e} \sim\right.$ $10^{6} \mathrm{~cm}^{-3}$ ) would suppress lines such as [O III] $\lambda 5007$ or [N II] $\lambda 6584$, without significantly affecting the strength of $[\mathrm{O} \mathrm{III}] \lambda 4363$ or $[\mathrm{N} \mathrm{II}] \lambda 5755$, so that incorrectly high electron temperatures would be derived from the usual diagnostic flux ratios. An example where this mechanism might be at work is the LMC PN SMP 85 (N69), for which Dopita et al. (1994) needed a 2-component model to match the HST data. Their high-density component $\left(\mathrm{n}_{\mathrm{e}}=10^{6} \mathrm{~cm}^{-3}\right)$ preferentially contibuted flux in the $[O \mathrm{III}] \lambda 4363$ line, but not in the $\lambda 5007$ line.

Space does not permit a full discussion of the heavy-element abundance ratios ( $\mathrm{N} / \mathrm{O}, \mathrm{C} / \mathrm{O}$, etc.) that have been derived for Magellanic Cloud PN - the reader is referred to the recent reviews by Barlow (1991) and Dopita (1993). We can however make use of the fact that (a) the oxygen abundances of PN have not been altered during their evolution, and (b) abundance ratios (e.g. N/O) should be reliable, provided both abundances have been derived from collisionally excited lines or both have been derived from recombination lines (i.e. not from mixtures of forbidden and recombination lines). The AGB progenitors of planetary nebulae are expected to have converted much of their original carbon into nitrogen via the $\mathrm{CN}$-cycle. $\mathrm{A}$ comparison between the N/O ratios of non-Type I PN and the $(\mathrm{C}+\mathrm{N}) / \mathrm{O}$ ratios of $\mathrm{H}$ II regions in the same galaxy reveals that in the PN progenitors the efficiency of conversion of the original $\mathrm{C}$ into $\mathrm{N}$ was about $30 \%$ for Galactic PN, $50 \%$ for LMC PN and nearly $100 \%$ for SMC PN, in excellent agreement with the predictions of Becker \& Iben (1980) as a function of metallicity. Another important conclusion can 
be derived for both Galactic and Magellanic Cloud Type I PN. In all three galaxies it is found that the $\mathrm{N} / \mathrm{O}$ ratio of the Type I nebulae is always significantly larger than the $(\mathrm{C}+\mathrm{N}) / \mathrm{O}$ ratio of the $\mathrm{H}$ II regions in the same galaxy. Since the oxygen abundances of the Type I PN and the $\mathrm{H}$ II regions are the same, this implies that the enhanced nitrogen in Type I PN must be primary, i.e. primary carbon must definitely have been brought up by the 3rd dredge-up event and then converted to nitrogen by envelope-burning (via the $\mathrm{CN}$-cycle) at the base of the hydrogen envelope, as predicted for sufficiently massive stars by evolutionary models.

\section{References}

Aller, L.H., Keyes, C.D., Ross, J.E. and O'Mara, B.J.: 1981, MNRA S, 194, 613

Aller, L.H., Keyes, C.D., Maran, S.P., Gull, T.R., Michalitsianos, A.G. and Stecher, T.P.: 1987, $A_{p} J, 320,159$

Barlow, M.J.: 1991, in Proc. IAU Symp. No. 148, The Magellanic Clouds, ed. R. Haynes and D. Milne, Kluwer Academic Press, 291

Barlow, M.J., Liu, X-W., Clegg, R.E.S., Storey, P.J. and Danziger, J.: 1995, MNRAS, to be submitted

Becker, S.A. and Iben, I. Jr.: 1980, A pJ, 237, 129

Boroson, T.A. and Liebert, J.: 1989, $A$ p J, 339,844

de Freitas Pacheco, J.A., Barbuy, B., Costa, R.D.D. and Idiart, T.E.P.: 1993a, A EA, 271, 429

de Freitas Pacheco, J.A., Costa, R.D.D. and Maciel, W.J.: 1993b, $A \mathcal{E} A, 279,567$

Dopita, M.A.: 1993 in Proc. IAU Symp. No. 155, Planetary Nebulae, ed. R. Weinberger and A. Acker, Kluwer Academic Press, 433

Dopita, M.A. and Meatheringham, S.J.: 1991a, A pJ, 367, 115

Dopita, M.A. and Meatheringham, S.J.: $1991 \mathrm{~b}, A$ pJ, 377, 480

Dopita, M.A., et al.: 1994, A pJ, 426, 150

Dufour, R.J., Shields, G.A. and Talbot, R.J.: 1982, A pJ, 252, 461

Grevease, N. and Noels, A.: 1993, in Origin and Evolution of the Elements, ed. N. Prantzos, E. Vangioni-Flam and M. Casse, Cambridge University Press, 15

Henry, R.B.C.: 1989, MNRAS, 241, 453

Henry, R.B.C.: 1990, ApJ, 356, 229

Henry, R.B.C., Liebert, J. and Boroson, T.A.: 1989, A pJ, 339, 872

Jacoby, G.H. and Kaler, J.B.: 1993, ApJ, 417, 209

Kaler, J.B. and Jacoby, G.H., 1990, ApJ, 362, 491

Kaler, J.B. and Jacoby, G.H., 1991, ApJ, 382, 134

Kingsburgh, R.L. and Barlow, M.J.: 1994, MNRA $S$, in press

Liu, X-W., Storey, P.J., Barlow, M.J. and Clegg, R.E.S.: 1995, MNRAS, in press

Meatheringham, S.J. and Dopita, M.A.: $1991 \mathrm{a}$, A p JS, 75, 407

Meatheringham, S.J. and Dopita, M.A.: 1991b, A pJS, 76, 1085

Monk, D.J., Barlow, M.J. and Cleg8, R.E.S.: 1988, MNRAS, 234, 583

Pagel, B.E.J., Edmunds, M.G., Fosbury, R.A.E. and Webster, B.L.: 1978, MNRAS, 184, 569

Peimbert, M.: 1967, ApJ, 150, 825

Peimbert, M.: 1971, Bol. Obs. de Tonanzintla y Tacubaya, 6, 29

Peimbert, M., Torres-Peimbert, S. and Ruiz, M.T.: 1992, Rev. Mex. Astron. Astrofis., 24, 155

Peimbert, M., Storey, P.J. and Torres-Peimbert, S.: 1993, A pJ, 414, 626

Richer, M.G.: 1993, ApJ, 415, 240

Russell, S.C. and Dopita, M.A.: 1990, ApJS, 74, 93

Shaver, P.A., McGee, R.X., Newton, L.M., Danks, A.C. and Pottasch, S.R.: 1983, MNRAS, 204, 53

Vassiliadis, E., Dopits, M.A., Morgan D.H. and Bell, J.F. 1992, ApJS, 83, 87

Viegas, S.M. and Clegg, R.E.S.: 1994, MNRAS, in press

Walton, N.A., Barlow, M.J., Clegg, R.E.S. and Monk, D.J.: 1991, in Proc. LAU Symp. No. 148, The Magellanic Closds, ed. R. Haynes and D. Milne, Kluwer Academic Press, 334 